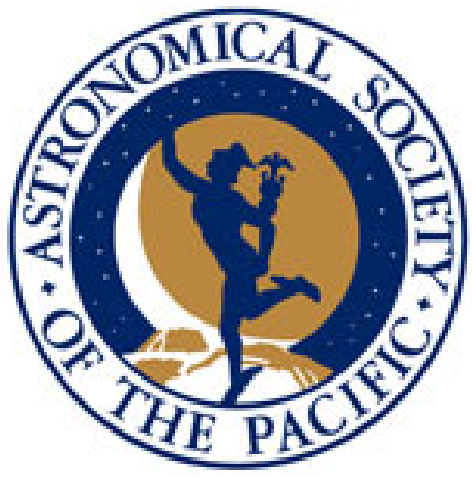

Self-Phase-Referenced Spectro-Interferometry on the Keck Interferometer Author(s): J. Woillez, R. Akeson, M. Colavita, J. Eisner, R. Millan-Gabet, J. Monnier, J.-U. Pott, S. Ragland, P. Wizinowich, M. Abajian, E. Appleby, B. Berkey, A. Cooper, C. Felizardo, J. Herstein, M. Hrynevych, D. Medeiros, D. Morrison, T. Panteleeva, B. Smith, K. Summers, K. Tsubota, C. Tyau, E. Wetherell

Reviewed work(s):

Source: Publications of the Astronomical Society of the Pacific, Vol. 124, No. 911 (January 2012), pp. 51-61

Published by: The University of Chicago Press on behalf of the Astronomical Society of the Pacific Stable URL: http://www.jstor.org/stable/10.1086/664075

Accessed: 02/03/2012 11:34

Your use of the JSTOR archive indicates your acceptance of the Terms \& Conditions of Use, available at http://www.jstor.org/page/info/about/policies/terms.jsp

JSTOR is a not-for-profit service that helps scholars, researchers, and students discover, use, and build upon a wide range of content in a trusted digital archive. We use information technology and tools to increase productivity and facilitate new forms of scholarship. For more information about JSTOR, please contact support@jstor.org. 


\title{
Self-Phase-Referenced Spectro-Interferometry on the Keck Interferometer
}

\author{
J. Woillez, ${ }^{1}$ R. Akeson, ${ }^{2}$ M. Colavita, ${ }^{3}$ J. Eisner, ${ }^{4}$ R. Millan-Gabet, ${ }^{2}$ J. Monnier, ${ }^{5}$ J.-U. Pott, ${ }^{6}$ S. Ragland, ${ }^{1}$ \\ P. Wizinowich, ${ }^{1}$ M. Abajian, ${ }^{2}$ E. Appleby, ${ }^{1}$ B. Berkey,,${ }^{1}$ A. Cooper,${ }^{1}$ C. Felizardo, ${ }^{2}$ J. Herstein, ${ }^{2}$ \\ M. Hrynevych, ${ }^{1}$ D. Medeiros, ${ }^{1}$ D. Morrison, ${ }^{1}$ T. Panteleeva,,${ }^{1}$ B. Smith,${ }^{1}$ K. Summers,${ }^{1}$ \\ K. Tsubota, ${ }^{1}$ C. Tyau, ${ }^{1}$ And E. Wetherell ${ }^{1}$ \\ Received 2011 July 1; accepted 2011 December 7; published 2012 January 30
}

\begin{abstract}
As part of the astrometric and phase-referenced astronomy (ASTRA) project, three new science modes are being developed for the Keck Interferometer that extend the science capabilities of this instrument to include higher spectral resolution, fainter magnitudes, and astrometry. We report on the successful implementation of the first of these science modes, the self-phase-referencing mode, which provides a $K$-band $(\lambda=2.2 \mu \mathrm{m})$ spectral resolution of $R \sim 1000$ on targets as faint as 7.8 mag with spatial resolution as fine as $\lambda / B=5$ mas in the $K$ band, with the $85 \mathrm{~m}$ interferometer baseline. This level of spectral resolution would not have been possible without a phase-referencing implementation extending the integration time limit imposed by atmospheric turbulence. For narrow spectral features, we demonstrate a precision of \pm 0.01 on the differential $V^{2}(\lambda)$, and $\pm 1.7 \mathrm{mrad}$ on the differential phase $\Phi(\lambda)$, equivalent to a differential astrometry precision of $\pm 1.45 \mu$ as. This new Keck Interferometer instrument is typically used to study the geometry and location of narrow spectral features at high angular resolution, referenced to a continuum. By simultaneously providing spectral and spatial information, the geometry of velocity fields (e.g., rotating disks, inflows, outflows, etc.) larger than $150 \mathrm{~km} \mathrm{~s}^{-1}$ can also be explored.
\end{abstract}

Online material: color figures

\section{INTRODUCTION}

The Keck Interferometer (KI) achieved first on-sky fringes with the two $10 \mathrm{~m}$ diameter Keck telescopes in 2001 (Colavita 2001), and the first science result was published in 2003 (Colavita et al. 2003). The $B=85 \mathrm{~m}$ baseline separation of the two telescopes produces an angular resolution of $\lambda / B=$ 5 mas at a wavelength $\lambda=2.2 \mu \mathrm{m}$ in the direction between the telescopes (the physical baseline has a position angle of $38^{\circ}$ east of north). The development of the KI was funded by NASA, to support future space missions. The interferometer is routinely used for both nulling (Colavita et al. 2009) and visibility science observations (Ragland et al. 2008).

In 2007, a proposal was funded by the NSF Major Research Instrumentation program to extend the capabilities of the KI, to address a broader range of science. The technical goals of this

\footnotetext{
${ }^{1}$ W.M. Keck Observatory, 65-1120 Mamalahoa Highway, Kamuela, HI 96743.

${ }^{2}$ NASA Exoplanet Science Institute, California Institute of Technology, Pasadena, CA 91125.

${ }^{3}$ Jet Propulsion Laboratory, California Institute of Technology, Pasadena, CA 91109.

${ }^{4}$ Steward Observatory, University of Arizona, Tucson, AZ 85721.

${ }^{5}$ University of Michigan, Ann Arbor, MI 48109.

${ }^{6}$ Max-Planck-Institut für Astronomie, Königstuhl 17, D-69117 Heidelberg, Germany.
}

ASTRA project (Woillez et al. 2010) upgrade program are to extend the interferometer's sensitivity through phase-referencing and to implement a narrow-angle astrometry mode (Shao \& Colavita 1992). The science topics driving the design of the new capabilities are (1) the study of the chemical composition and geometry of the inner parts of young stellar objects, (2) the measurements of the cores of active galactic nuclei, (3) an unbiased determination of the masses of known exoplanets, and (4) a probe of general relativity through high precision astrometric measurements of stars orbiting the massive black hole at the Galactic center. The driving goals (topics 3 and 4) of the ASTRA project are similar to those of the PRIMA astrometric facility (PRIMA; Quirrenbach et al. 1998; Delplancke et al. 2006) and of the GRAVITY project (Eisenhauer et al. 2005), both on VLTI.

The upgrade of an existing instrument routinely used for science observations requires an implementation strategy that minimizes the impact on the operational system. A threestep approach, self-phase-referencing (SPR), dual-field phasereferencing (DFPR), and astrometry (AST), that gradually develops the full ASTRA functionality was therefore chosen. The three modes were designed to each provide improved science capabilities, while building on the infrastructure and lessons learned provided by the previous mode. In this article we report on the implementation results from the first SPR step, which enables science topic1 from the list given above. 
The SPR instrument combines the 5 mas angular resolution delivered by the Keck Interferometer baseline, to an $R \sim 1000$ $K$-band spectrometer. The increased spectral resolution is made possible, without a drastic limiting magnitude degradation, by a fringe tracker stabilizing the fringe phase and allowing long exposure to be performed by the spectrometer.

The SPR instrument is not the first spectro-interferometer: the technique was first demonstrated in the visible with the GI2T interferometer (Mourard et al. 1989; Vakili et al. 1997, 1998; Berio et al. 1999). Application to the infrared followed, but without fringe tracking and therefore with limited sensitivity. The first phased-referenced observations (Le Bouquin et al. 2008) were performed on the VLTI by the AMBER spectrometer (Petrov et al. 2007) in the $K$ band, with the fringe tracker FINITO (Gai et al. 2004) stabilizing the fringes in $H$, this is the closest match to the SPR instrument. A self-phase-referencing mode has also been used by the visible interferometric spectrograph VEGA (Mourard et al. 2009) on CHARA, stabilized by the group-delay trackers CLIMB (Sturmann et al. 2010) or MIRC (Monnier et al. 2008). However, using a visible photoncounting camera, the fringe stabilization need is related to a measurement precision requirement in the short integration period, rather than to a limiting magnitude requirement as in the infrared.

Section 2 provides a description of the SPR instrument and how it fits into the existing interferometer. Section 3 details the limiting magnitude error budget, as set by the fast fringe tracker. Section 4 presents the performance of the two observables delivered by SPR: square visibility $V^{2}(\lambda)$ and spectral differential phase $\Phi(\lambda)$.

\section{INSTRUMENT DESCRIPTION}

\subsection{Keck Interferometer Overview}

In visibility mode, the optical path from each telescope includes a $10 \mathrm{~m}$ Keck telescope, a natural guide star adaptive optics (AO) system (Wizinowich et al. 2000), an interferometer module that takes collimated light from the AO system and sends it down a coudé train into the telescope pier, beam transport optics that transport the light into the interferometer basement, a long delay line that is positioned to minimize the optical path difference between the two telescopes for a given position on the sky, fast delay lines that maintain zero optical path difference and dither by one wavelength around it, and an angle tracker that measures and corrects the tip-tilt wavefront errors. The beams from the two telescopes are interfered in the KI fringe tracker (Vasisht et al. 2003) at a beam splitter and the combined light is injected into two single-mode fibers whose outputs are imaged onto a fast near-infrared camera (i.e., the fringe tracker camera). The entire $K$ (or $H$ ) band from one fiber is imaged onto a single pixel of the Rockwell $\mathrm{HgCdTe}$ Hawaii array to measure the fringe phase and visibility. The $K$ (or $H$ ) band light from the second fiber is dispersed over a few pixels (typically 5) to provide a group-delay estimate. The fringe tracking algorithm makes use of both the phase and group delays to control the delay-line position to stabilize the fringes (Colavita et al. 2010). The fringe measurement approach uses a onewavelength scan over the fringe implemented by the delay line; the fringe tracker is read out after each quarter-wave of pathlength change. The $V^{2}$ and phase observables are computed from these four quarter-wave samples.

The SPR implementation builds upon the existing visibility and nuller infrastructure with two main modifications: a reorganization of the interferometer beam train to implement an optical path difference (OPD) stabilization architecture (described in $\S 2.2$ ) to feed a new science beam combiner and the implementation of a $R \sim 1000$ spectrometer (described in $\S 2.3$ ).

\subsection{Phase Stabilization Architecture}

The optical path stabilization architecture for the SPR instrument is largely inspired from the Keck Nuller architecture (Colavita et al. 2006, 2008), where $K / N$ dichroic beam splitters, located just before the fast delay lines, separate the transmitted $K$-band light, used to stabilize the OPD, from the reflected $N$-band light, directed to the Nuller itself. In the $K$ band these beam splitters transmit $\sim 20 \%$ of the light and reflect $\sim 55 \%$ and can be used for a $K$-band to $K$-band phase stabilization scheme, despite not being optimized for this purpose. Ideally, we would have used a dedicated splitter optimized for the best limiting magnitude: without the $\sim 25 \%$ losses and sending most of the light to the fringe tracker. However, we accepted the low transmission to the fringe tracker in order to minimize cost and impact on the existing interferometer setup. The impact of the $K / N$ splitters on the limiting magnitude error budget is given in $\S 3$.

The conceptual layout of the SPR instrument is presented in Figure 1. On the transmitted side, a fringe tracking setup identical to the Nuller is used to stabilize the OPD for a new $K$-band spectrometer located on the reflected side of the $K / N$ dichroic. The reconfiguration to the SPR instrument is achieved by rerouting the beam at the output of the reflected-side delay lines (the spectrometer delay lines), by means of retractable mirrors, toward the spectrometer. Figure 1 also presents the OPD control diagram for the SPR instrument. The system runs at a frame rate of $250 \mathrm{~Hz}$ using a sliding-window approach, providing delay updates at $1250 \mathrm{~Hz}$ (Colavita et al. 2010). These updates are used to close a feedback loop to the fringe tracker delay lines and also to send feedforward commands to the spectrometer delay lines. The use of delay lines dedicated to the spectrometer provides a higher OPD error rejection factor than a pure feedback system and attenuates disturbances below about $40 \mathrm{~Hz}$ (see Colavita et al. 2010, Figs 5 and 6). In addition to the feedforward command to the spectrometer delay lines, the fast fringe tracker sends a hold command to the spectrometer when it is not locked on the white light fringe. The purpose of this hold is twofold. First, it prevents the spectrometer from searching 




FIG. 1.-Schematic of the SPR optical setup, including the control loops involving the fringe tracker and the spectrometer. The $K / N$ splitters send $20 \%$ of the light collected by the two Keck telescopes to the fringe tracker and $55 \%$ to the spectrometer. The fringe tracker not only follows the interference fringes by controlling its own delay lines in a feedback loop (gain of less than 1 for stability), but also sends a correction to the spectrometer delay lines (gain equal to 1 , no stability requirement), thereby stabilizing the fringes for the spectrometer to perform long integrations. The spectrometer controls its own delay lines in a low-bandwidth feedback loop, as long as it is not held by the fringe tracker (something that happens when the fringe tracker is not properly locked on the central fringe). Note that the visibility mode consists simply of the fringe tracker delay lines and fringe tracker (without the $K / N$ splitters in the beam). See the electronic edition of the PASP for a color version of this figure.

and tracking on its own white light fringe when the OPD is not properly stabilized. Second, it also prevents the data recorded by the spectrometer from being used by the data processing pipeline and therefore delivers a highly stable fringe contrast.

\subsection{Spectrometer Beam Combiner and Camera}

From the second pair of delay lines, the differential-pistonstabilized light is directed to a beam combiner that is identical to the fringe tracker beam combiner. This combiner is based on a free-space 50:50 beam splitter and compensator. The two interferometric outputs are injected into infrared single-mode fibers that feed the science camera. This camera is different from the fringe tracker camera (Vasisht et al. 2003) by only one element: the 5 pixel dispersion prism, used to compute the group delay for the fringe tracker, is replaced by an $R \sim 1000$ dispersive element. This grism has a vendor-measured first-order transmission efficiency varying from $53 \%$ at $2.0 \mu \mathrm{m}$ to $36 \%$ at $2.4 \mu \mathrm{m}$ (44\% on average). Vignetting within the unmodified camera limits the full width at half-maximum spectral coverage to $233 \mathrm{~nm}$ contained within the $K$ band, compatible with the simultaneous observation of the $\operatorname{Br} \gamma(2.166 \mu \mathrm{m})$ and $\mathrm{CO}$ band head
(2.293 $\mu \mathrm{m}, 2.323 \mu \mathrm{m}$, and $2.352 \mu \mathrm{m})$ emission lines. The spectrometer is operated in a similar way to the fringe tracker, using a four-bin ramp-scanning algorithm, but at a much slower rate. An integration time on the order of a few seconds (compared with the fringe tracker rate of 200 to $250 \mathrm{~Hz}$ ) is sufficient to recover the signal-to-noise ratio $(\mathrm{S} / \mathrm{N})$ loss per spectral channel, resulting from the higher spectral resolution and the lower grism throughput. The nondispersed channel is attenuated to prevent saturation at low frame rates. Using both the dispersed and nondispersed channels, the spectrometer is able to measure the group and phase delays and to control its delay lines at a low bandwidth to center the fringe measurements on zero OPD, compensating for slow thermal drifts with respect to the fast fringe tracker. Finally, as mentioned in $\S 2.2$, the spectrometer servos with its own delay lines only when not held by the fast fringe tracker.

The wavelength of each pixel in the spectrometer has been measured using a Fourier transform spectrometer (FTS) setup. The fast delay lines scan through a fringe packet obtained from an internal source reflected off retroreflectors. For each pixel, the location of the peak in the Fourier transform of the detected signal gives the wavelength. The wavelengths obtained from this FTS method have been compared with the emission lines of a neon lamp (see Fig. 2), illuminating the entrance fiber to the fringe tracker camera. The agreement between the FTS-generated wavelength table and the known wavelength of the neon lamp is better than $\pm 0.1 \mathrm{~nm}^{7}$ The wavelength table obtained from the FTS method has then been linearly shifted to have the wavelength of the brightest spectral line (at $2.364 \mu \mathrm{m}$ ) correspond to one specific pixel of the detector. Before each observing night, the spectrometer is realigned by adjusting the location of that same spectral line to the same reference pixel. With an alignment accuracy of one-tenth of a pixel, the wavelength table was measured to be accurate to $\pm 0.1 \mathrm{~nm}$. Finally, the stability of the spectrometer throughout a night has been measured to be stable to within 0.02 pixels, which amounts to $0.025 \mathrm{~nm}$.

The spectrometer suffers from being undersampled by a factor of 2 in the spectral direction. This is a consequence of the original spectrometer design, which was optimized for sensitivity: when no dispersing element is inserted, the optics inside the camera are dimensioned to concentrate the light from each feeding fiber onto one single pixel. Since spectral Nyquist sampling is reached at $R=1000$, aliasing effects may need to be taken into account in the data analysis, between the Nyquist sampling and an optical spectral width of at least $R \sim 2000$ (1 pixel). In addition, the optical spectral resolution decreases at the edges of the spectral bandpass due to diffraction caused by internal vignetting (decrease by a factor of 2 from $R \sim 2000$ at $50 \%$ transmission; see Fig. 3). Taking all these considerations into account, we cannot advertise a spectral resolution higher than $R \sim 1000$.

\footnotetext{
${ }^{7}$ All $\pm x$ notations have a 1- $\sigma$ meaning.
} 




FIG. 2.-Measured neon lamp spectrum, with the most intense emission lines identified with the NIST Atomic Spectra Database (http://physics.nist.gov/asd). The emission line used for the alignment of the spectrometer is at $\lambda=2253.65 \mu \mathrm{m}$. See the electronic edition of the PASP for a color version of this figure.

\subsection{Observing Sequence}

The sequencing of the observations needed to calibrate the data for the SPR instrument is directly adapted from the visibilitymode sequence (Colavita et al. 2003). The most noticeable difference is the initial target acquisition step, which optimizes the pointing of the observed object onto the spectrometer fibers. This step is performed in a reasonable amount of time by temporarily increasing the frame rate of the spectrometer to $200 \mathrm{~Hz}$ and removing the attenuation on the nondispersed channel. A fastscanning mirror is used to explore the field at the nondispersed single-mode fiber input of the camera and to optimize the injection. The spectrometer is then reverted to a low frame rate and an attenuated nondispersed channel, before carrying out the observations. This operation also optimizes the injection onto the spectral fiber, since it is co-aligned with the nondispersed fiber.

For the data acquisition, collections of individual fringe measurements are interleaved with single-telescope flux measurements used to calibrate out the flux-ratio-dependent contrast loss. This data collection operation is then repeated on a calibrator target, ${ }^{8}$ in order to remove instrumental and atmospheric effects from the science target observables. The square visibility $V^{2}$ of the science target is normalized with a ratio to the calibrator square visibility, whereas the differential phase $\Phi$ of the calibrator is subtracted from the differential phase of the science target: ${ }^{9}$

\footnotetext{
${ }^{8} \mathrm{~A}$ target with a small diameter and a well-known $V^{2}(\lambda)_{\text {cal }}$ or $\Phi(\lambda)_{\text {cal }}$, usually without spectral features.

${ }^{9}$ See nbCalib (http://nexsci.caltech.edu/software/V2calib/) for the calibration of $V^{2}(\lambda)$. The calibration of $\Phi(\lambda)$ is left to the user to perform, following eq. (2).
}
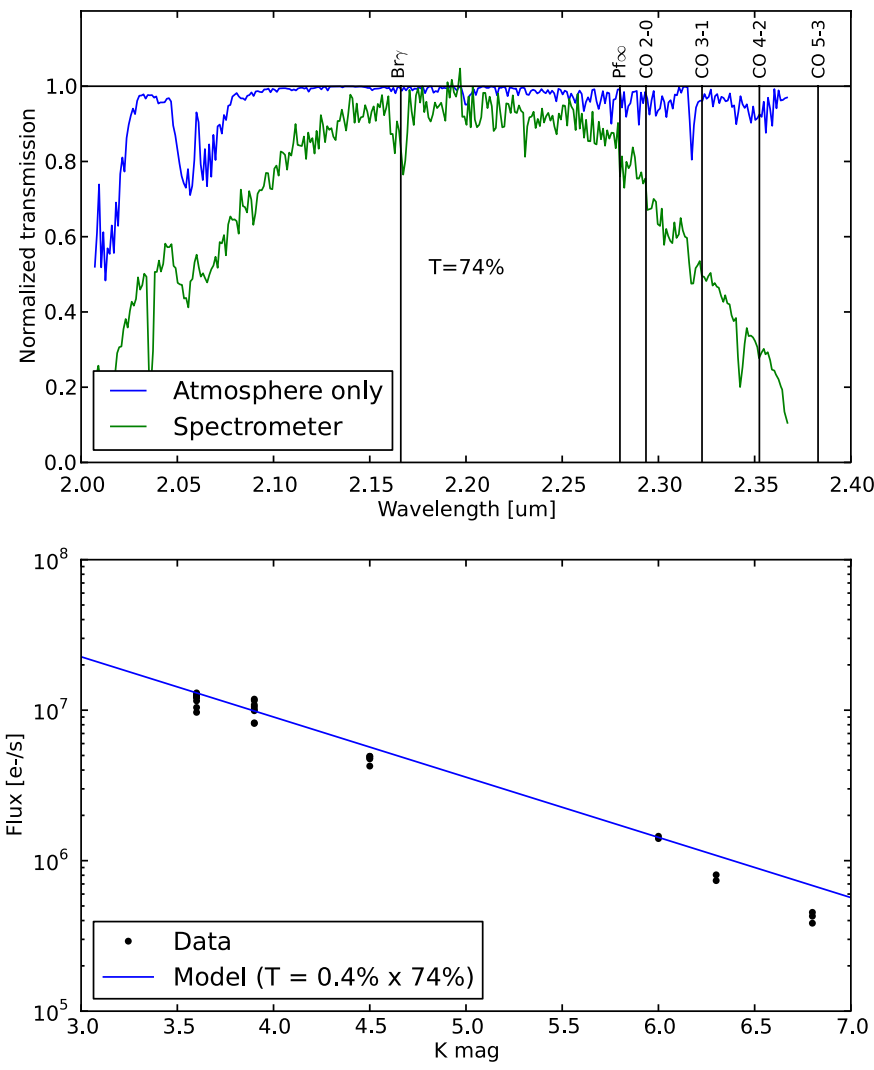

FIG. 3.-Top: Normalized spectral transmission of the spectrometer, compared with a typical Mauna Kea atmospheric transmission profile (air mass of 1 , water vapor column of $1 \mathrm{~mm}$ ). The locations of $\operatorname{Br} \gamma, \operatorname{Pf} \infty$, and some of the $\mathrm{CO}$ band heads are given to illustrate some of the spectral features observable with the instrument. The error budget presented in Table 1 is given for the peak transmission of around $2.2 \mu \mathrm{m}$. The transmission losses at the edges of the wavelength range are caused by vignetting internal to the camera and amounts to a broadband transmission vs. peak transmission of $T=77 \%$ over a bandwidth of $350 \mathrm{~nm}(2.0 \mu \mathrm{m}$ to $2.35 \mu \mathrm{m})$. Bottom: Flux detected on the spectral side of the spectrometer vs. $K$ magnitude. The overlaid transmission model ( $T=0.4 \%$ from the Table 1 error budget, combined with the spectral transmission of $T=77 \%$ ) is in agreement with measurements. See the electronic edition of the PASP for a color version of this figure.

$$
\begin{gathered}
V^{2}(\lambda)_{\mathrm{sci}}=\frac{V^{2}(\lambda)_{\text {sci,meas }}}{V^{2}(\lambda)_{\text {cal,meas }}} \cdot V^{2}(\lambda)_{\mathrm{cal}}, \\
\Phi(\lambda)_{\mathrm{sci}}=\left[\Phi(\lambda)_{\text {sci,meas }}-\Phi(\lambda)_{\text {cal,meas }}\right]+\Phi(\lambda)_{\mathrm{cal}} .
\end{gathered}
$$

These are standard calibration procedures in optical longbaseline interferometry (see, e.g., Perrin 2003 and Millour et al. 2007 for $V^{2}(\lambda)$ and $\Phi(\lambda)$, respectively). A summary of the observing sequence, including its timing, is given in Figure 4.

Especially for fainter objects, the measurements on the nondispersed port of the spectrometer are not as accurate as those achieved by the classical $V^{2}$ mode and should therefore not be 


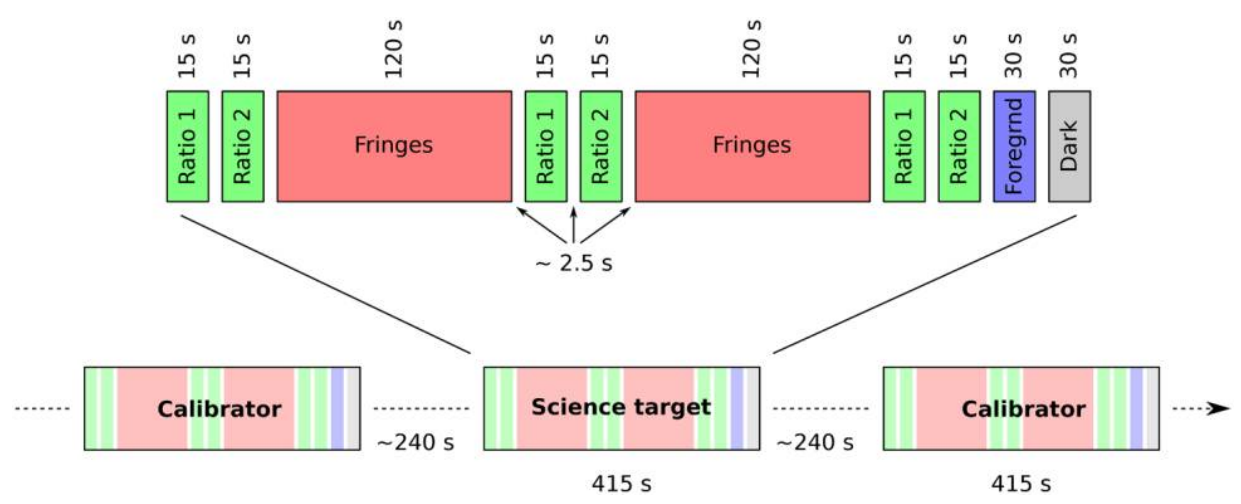

FIG. 4.-Summary of a typical observing sequence performed simultaneously on the fringe tracker and the spectrometer. Each science target observation is bracketed with observations of calibrators. Each observation on a science target or a calibrator is composed of a succession of ratios (independent flux measurements from each telescope) and fringe collections, concluding with a foreground (total flux, off-fringe) and a dark measurement. The timing of each phase is given in the figure. Each individual observation takes about $240 \mathrm{~s}$ of acquisition overhead (including telescope slew, adaptive optics acquisition, beam train acquisition, and pointing optimization onto the fringe tracker), followed by $415 \mathrm{~s}$ of data collection (including a $2.5 \mathrm{~s}$ gap between each step). A calibrated observation, which includes the observation of one science target paired with a calibrator, takes about $2 \times 11$ minutes (about 2.7 calibrated observations per hour). See the electronic edition of the $P A S P$ for a color version of this figure.

used. The two main reasons are the attenuation of the nondispersed port required by the longer integration and the increased contribution of the thermal background over longer integration periods. Instead, a broadband $V^{2}$ measurement is available simultaneously from the fringe tracker (calibration sequences are performed on the fringe tracker in parallel with the spectrometer calibration sequences) with an accuracy comparable with what the typical $V^{2}$ mode provides. The only performance difference with $V^{2}$ is the sensitivity reduction caused by the $20 \%$ transmission of the $K / N$ splitters.

\subsection{Data Reduction and Observables}

The data reduction pipeline (Kvis; Colavita 1999; Akeson \& Millan-Gabet 2005) is unchanged, except for the addition of a differential-phase $\Phi(\lambda)$ data product, and the rejection of data when the primary fringe tracker is not properly locked on fringes (following the hold command described earlier). For this differential-phase product, the phasors from each spectral channel, already calculated and calibrated at the level of a single fringe measurement, are first derotated by the white light phase and coherently summed over a first timescale ("frame" timescale). The spectral phase is computed at that same timescale. Then, a second level of averaging (to the "block" timescale), this time incoherently summed, is applied to the spectral phase. ${ }^{10}$ The end result is a differential-phase measurement reported at the same rate as the visibility measurements. The two final observables are the square visibility and the differential phase, each as a function of wavelength.

\footnotetext{
${ }^{10}$ Both frame and block timescales are defined in the Kvis manual (Akeson \& Millan-Gabet 2005).
}

Special care must be taken in the interpretation of the phase. Neither the absolute phase nor its first derivative with respect to the wave number (i.e., the residual group delay) are astrophysically significant (e.g., Schmitt et al. 2009; Millour et al. 2007). In addition, dispersion fluctuations, caused by (primarily) atmospheric water vapor turbulence, and quasistatic air dispersion, attributable to the use of nonevacuated delay lines, make broadband differential-phase features harder to detect and interpret. For these reasons, we chose to focus this study on relatively narrow spectral features, as detailed in $\S 4$.

\section{ERROR BUDGET AND LIMITING MAGNITUDE}

Table 1 gives a summary of the error budget for the SPR fringe tracker and spectrometer. The error budget in the $V^{2}$ configuration is also given as a reference, emphasizing the specific aspects of the SPR instrument. Between the $V^{2}$ and SPR modes, the addition of the $K / N$ splitters lowers the transmission to the fringe tracker by a factor of $5(\Delta K=-1.75 \mathrm{mag})$. With the current $V^{2}$ limiting magnitude of $K=9.55$ for the K5 mode at $250 \mathrm{~Hz}$, the SPR limiting magnitude becomes $K=7.8$. This limit has been verified on sky.

For both $V^{2}$ and SPR configurations, the limiting magnitude is set by the fringe tracker phase $\mathrm{S} / \mathrm{N}$ (Walkup \& Goodman 1973) of 10 for a single fringe measurement. This requirement corresponds to a stable fringe tracker lock. ${ }^{11}$ The phase $\mathrm{S} / \mathrm{N}$ estimation for the fringe tracker is based on the total flux (100\% observable transmission, see Table 1) from the two beamcombiner outputs, detected on 6 pixels (1 broadband pixel and 5 spectral pixels). For the spectrometer we are instead interested

\footnotetext{
${ }^{11}$ Some of the observations in Eisner et al. (2010) were performed up to 0.7 mag past the $K=7.8$ limit, but with a much degraded fringe tracking performance.
} 
TABLE 1

Self-Phase-Referencing ERror Budget Summary

\begin{tabular}{|c|c|c|c|c|}
\hline Quantity & $V^{2}$ Fringe tracker ${ }^{\mathrm{a}}$ & SPR Fringe tracker ${ }^{\mathrm{b}}$ & SPR Spectrometer $^{\mathrm{c}}$ & Unit \\
\hline Flux above two telescopes for $K=10, F_{\text {in }} \ldots$. & $2.25 \times 10^{4}$ & $2.25 \times 10^{4}$ & $2.25 \times 10^{4}$ & Photon $\mathrm{ms}^{-1}$ \\
\hline Static transmission & $5.66 \%$ & $5.66 \%$ & $5.66 \%$ & $\ldots$ \\
\hline Turbulent transmission $\ldots . .$. & $30.8 \%$ & $30.8 \%$ & $30.8 \%$ & $\ldots$ \\
\hline$K / N$ splitter $\ldots \ldots \ldots \ldots \ldots \ldots$ & $\ldots$ & $20 \%$ & $55 \%$ & $\ldots$ \\
\hline Grism transmission $\ldots \ldots \ldots$ & $\ldots$ & $\ldots$ & $45 \%$ & $\ldots$ \\
\hline Total transmission, $\% T \quad \ldots \ldots \ldots \ldots \ldots \ldots \ldots \ldots$ & $1.74 \%$ & $0.35 \%$ & $0.43 \%$ & $\ldots$ \\
\hline Flux at combiner outputs, $F_{\text {out }} \quad \ldots \ldots \ldots \ldots \ldots$ & 391.5 & 78.75 & 96.75 & $e^{-} \mathrm{ms}^{-1}$ \\
\hline 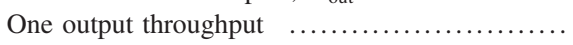 & 50.9 & 10.2 & 12.6 & $\mathrm{DN} \mathrm{ms}{ }^{-1}$ \\
\hline Observable transmission, $\gamma \ldots \ldots \ldots \ldots \ldots \ldots$ & $100 \%$ & $100 \%$ & $0.16 \%$ & $\ldots$ \\
\hline$K$ magnitude $\quad \ldots \ldots \ldots \ldots \ldots \ldots \ldots \ldots \ldots \ldots \ldots \ldots \ldots \ldots \ldots \ldots$ & 9.55 & 7.80 & 7.80 & mag \\
\hline Integration time, $t \quad \ldots \ldots \ldots \ldots \ldots \ldots \ldots \ldots \ldots$ & 0.004 & 0.004 & 1.0 & $\mathrm{~s}$ \\
\hline Electrons per observable, $N \quad \ldots \ldots \ldots \ldots \ldots \ldots$ & 2375 & 2381 & 1112 & $e^{-}$ \\
\hline Residual piston, $\sigma_{\mathrm{ft}} \ldots \ldots \ldots \ldots \ldots \ldots \ldots \ldots \ldots$ & 165 & 165 & 165 & $\mathrm{~nm}$ \\
\hline Differential piston, $\sigma_{\text {spectro }} \ldots \ldots \ldots \ldots \ldots \ldots \ldots$ & $\ldots$ & $\ldots$ & 200 & $\mathrm{~nm}$ \\
\hline Fringe contrast, $\mu^{2} \quad \ldots \ldots \ldots \ldots \ldots \ldots$ & 0.80 & 0.80 & 0.58 & $\ldots$ \\
\hline 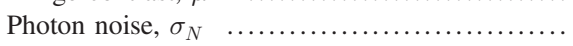 & 49 & 49 & 33 & $e^{-}$ \\
\hline Background noise, $\sigma_{B} \quad \ldots \ldots \ldots \ldots \ldots \ldots \ldots \ldots$ & 4 & 5 & 3 & $e^{-}$ \\
\hline 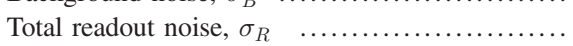 & 49 & 49 & 10 & $e^{-}$ \\
\hline 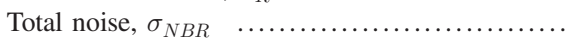 & 69.0 & 69.0 & 35.0 & $e^{-}$ \\
\hline 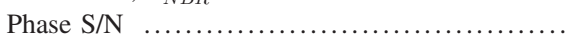 & 17.5 & 17.5 & 11.7 & $\ldots$ \\
\hline Phase error rms, $\sigma_{\Phi} \quad \ldots \ldots \ldots \ldots \ldots \ldots$ & 3.3 & 3.3 & 4.9 & deg \\
\hline
\end{tabular}

${ }^{\text {a }}$ The fringe tracker error budget in $V^{2}$ mode is presented for its advertised $K=7.8$ band limiting magnitude.

${ }^{\mathrm{b}}$ The error budget for the fringe tracker in SPR mode is given for $K=7.8$, which takes into account the sensitivity loss from the $20 \%$ transmissive $K / N$ splitter.

${ }^{\mathrm{c}}$ The error budget for the spectrometer is given for a single spectral pixel, an identical $K=7.8$ limiting magnitude, and an integration time of $1 \mathrm{~s}$, which offsets transmission losses, differential piston with the fringe tracker, and narrow spectral bandwidth. Details on the error budget calculations are given in Appendix A.

in the performance of a single spectral channel. The phase $\mathrm{S} / \mathrm{N}$ estimation is therefore different: one single $1.1 \mathrm{~nm}$ wide spectral channel from one beam-combiner output $(0.16 \%$ observable transmission for one of the 330 pixels covering the $K$ band). For optimal operation, the spectrometer integration time should be long enough to reach a photon-noise-limited regime, but still short enough to perform many fringe measurements. In Table 1, a $1 \mathrm{~s}$ integration time at the $K=7.8$ limiting magnitude provides such a compromise. For brighter objects, the spectrometer integration time should be decreased accordingly. The transmission model for the spectrometer has been verified on sky (bottom of Fig. 3), taking into account the transmission profile of the spectrometer camera (top of Fig. 3).

Additional details on the fringe tracker performance are available in Colavita et al. (2010). The remainder of this article focuses on the performance of the spectrometer observables.

\section{PERFORMANCE}

\subsection{Square Visibility: $V^{2}(\lambda)$}

In order to assess the accuracy of the visibility measurements, actual measurements are compared with reliable predictions. Binary stars of known characteristics are used, since they can provide good coverage of the visibility range while being accurately modeled with only a few simple parameters: position angle and separation (from orbital parameters), flux ratio, and stellar diameters.

On UT 2008 Novermber 16, we observed the well-known binary HD 9939, previously studied with the Palomar Testbed Interferometer (Boden et al. 2006). Due to scheduling and weather constraints the target could only be observed for about an hour, leading to three independent $V^{2}$ measurements at slightly different spatial frequencies. The observation parameters and dates are given in Tables 2 and 3 . The predicted $V^{2}$ change among measurements is only between 0.02 and 0.03 . The comparison between the measurements and the models is presented in Figure 5.

TABLE 2

ObSERVATION PARAMETERS OF BINARY STAR HD 9939

\begin{tabular}{cccc}
\hline \hline \multicolumn{1}{c}{ Parameter } & \multicolumn{2}{c}{ Primary } & Secondary \\
\hline Diameter (mas) & $\ldots \ldots$ & $0.451 \pm 0.018$ & $0.196 \pm 0.017$ \\
Luminosity $\left(L_{\odot}\right)$ & $\ldots \ldots$ & $2.451 \pm 0.041$ & $0.424 \pm 0.023$ \\
\hline
\end{tabular}

Note.-Used to validate the accuracy of the $V^{2}(\lambda)$ observable. Diameter and luminosity are directly from Boden et al. (2006). 
TABLE 3

Observation Dates of BinARY Star HD 9939

\begin{tabular}{cccc}
\hline \hline $\begin{array}{c}\text { Julian date } \\
\text { (day) }\end{array}$ & & $\begin{array}{c}\text { R.A. offset } \\
\text { (mas) }\end{array}$ & $\begin{array}{c}\text { Decl. offset } \\
\text { (mas) }\end{array}$ \\
\hline $2,454,786.879649$ & $\ldots \ldots \ldots$ & 2.9075 & 1.9049 \\
$2,454,786.901906$ & $\ldots \ldots \ldots$ & 2.9300 & 1.8977 \\
$2,454,786.916016$ & $\ldots \ldots \ldots$ & 2.9444 & 1.8930 \\
\hline
\end{tabular}

NoTE.-Component separations at the observation dates are computed from the orbit parameters given in Boden et al. (2006).

Figure 5 shows two important properties of the achievable precision of a differential $V^{2}$ measurement with the SPR instrument. First, the broadband calibration precision is shown to be better than \pm 0.02 (we still recommend using the fringe tracker for broadband measurements). Note that the last scan shown in Figure 5 could not be bracketed by calibrator measurements during the observations. We attribute the decreased absolute calibration precision of this scan to this nonoptimum calibration. Accordingly, the center of the histogram in the lower right panel of Figure 5 is further away from zero than for the two other scans. Second, a significantly better precision is achieved on the differential $V^{2}(\lambda)$. The widths of the histograms in the right panels show a differential precision of \pm 0.01 for each scan, which is important in order to be able to detect shallow features in the visibility spectrum and to properly calibrate the visibility contribution of an emission- or absorption-line feature against the continuum (Pott et al. 2010). Note that the width of

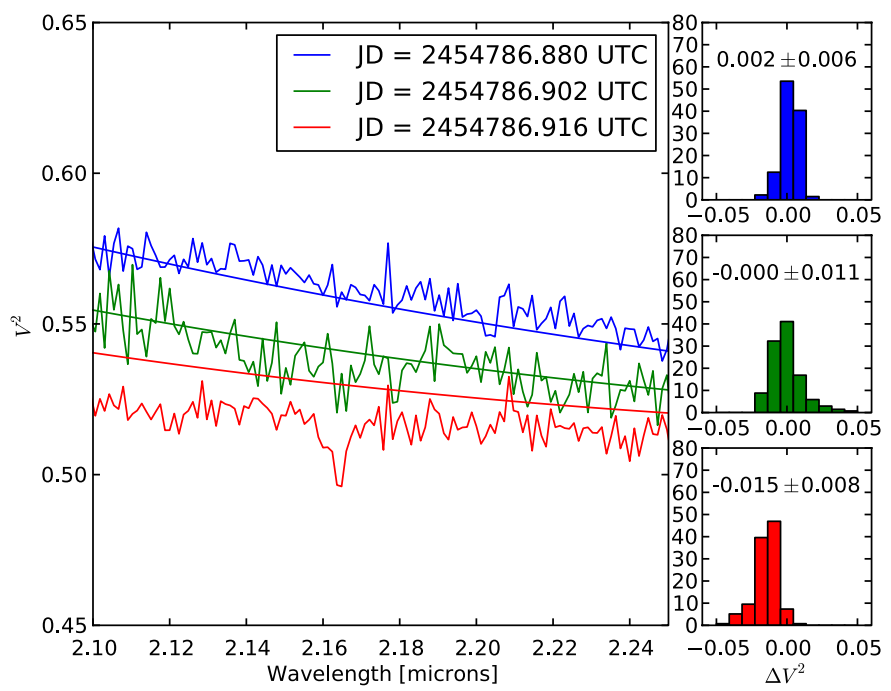

FIG. 5.-Left: Measured $V^{2}$ for the binary star HD 9939 compared with the model at three epochs, on UT 2008 November 16. Right: Histogram of the deviation $\Delta V^{2}$ from the model for each measurement. The absolute precision is better than \pm 0.02 , and the differential precision is better than \pm 0.01 . See the electronic edition of the PASP for a color version of this figure. the histogram of the last scan is equally narrow, i.e., the differential precision is not affected by the suboptimum calibration.

\subsection{Differential Phase: $\Phi(\lambda)$}

As stated in $\S 2.5$, the SPR instrument is not designed to measure broadband differential-phase signatures. The dispersion of dry air through the interferometer introduces a static differential-phase bias proportional to the total optical path difference (amount of additional air in one arm of the interferometer), whereas water vapor turbulence adds a variable bias. An illustration of these two effects is presented in Figure 6, where the phase curvature $\partial^{2} \Phi / \partial \sigma^{2}$ is given as a function of the optical path difference. The linear fit corresponds to the static dry air term, and the spread of the data around that fit is associated with water vapor turbulence. Note that this phase curvature versus optical path difference is the next phase derivative of the dispersion effect shown in Figure 2 of Akeson et al. (2000).

To assess the precision of the $\Phi(\lambda)$ observable, we studied its noise behavior on calibrator stars expected to be without significant differential-phase signatures. Specifically, we wanted to link the precision of a single spectral channel to a precision estimated across all the channels of the instrument. We therefore had to remove residual dispersion effects better than what the standard procedure (eq. [2], § 2.5) achieves. The metric that we used is the standard deviation over the wavelength of the differential phase, averaged over consecutive measurements, with a best-fit seventh-order polynomial $P_{7}(\lambda)$ removed:



FIG. 6.-Differential-phase curvature vs. optical delay. With delay lines not in vacuum, differential phase is impacted by the dispersion of air though the interferometer. Most of the contribution comes from a static term contained in the linear fit shown. The departure from that linear fit can be associated with water vapor turbulence. Both effects would have to be addressed to make a broadband differential-phase feature of astrophysical origin observable. See the electronic edition of the PASP for a color version of this figure. 

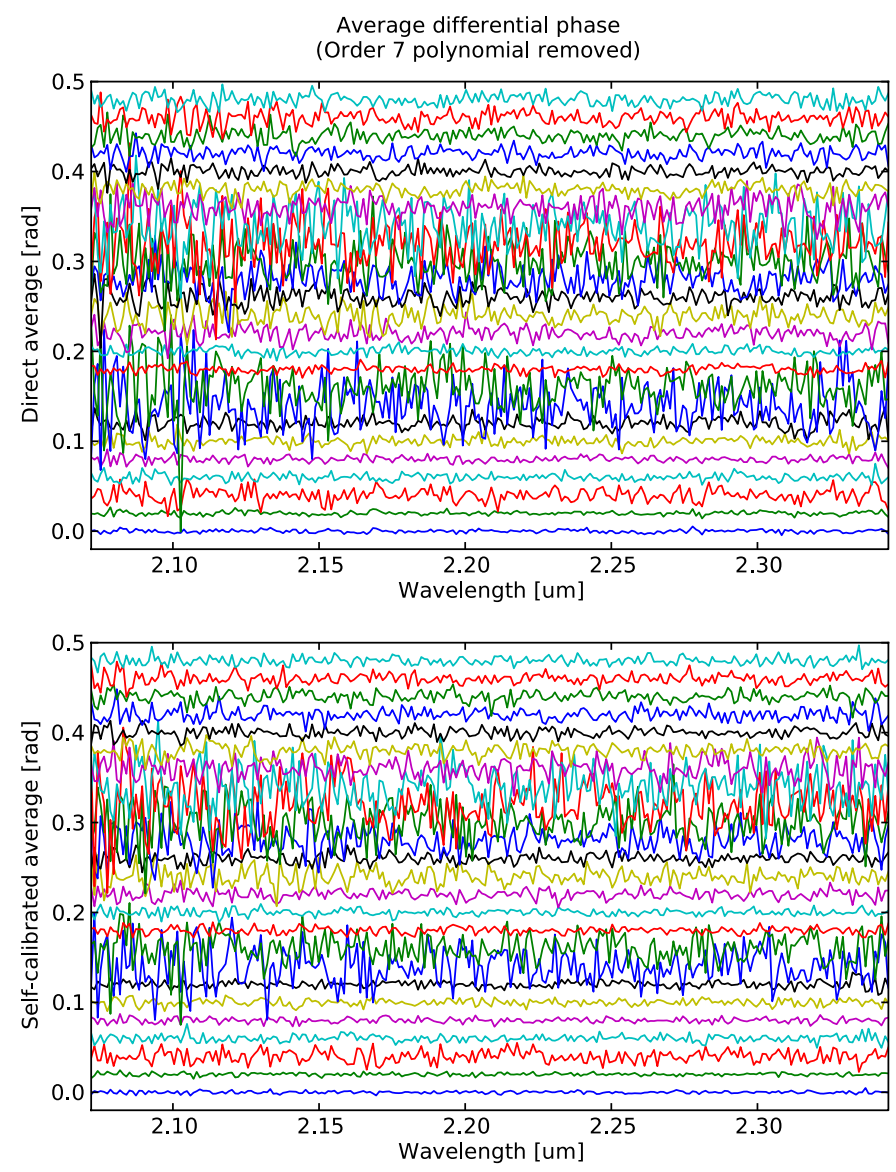

FIG. 7.-Top: Direct average differential phase for all the targets observed on UT 2009 July 17. For clarity, each measurement is shifted by 0.02 rad from the previous one. Bottom: Self-calibrated average differential phase, for the same data set, shifted as well. The differential-phase standard deviations of Fig. 8 are estimated from the plots here. The direct average is intended to show potential differential-phase biases, in comparison with the self-calibrated average intended to only show photon-noise effects. See the electronic edition of the $P A S P$ for a color version of this figure.

$$
\sigma_{\lambda}\left[\left\langle\Phi_{n}(\lambda)\right\rangle_{n}-P_{7}(\lambda)\right]
$$

Choosing 7 as the order of the polynomial is a somewhat arbitrary ${ }^{12}$ compromise between discarding the unwanted broadband signatures and preserving the narrowband features. For observations performed on UT 2009 July 17, the individual average differential phase with polynomial removed are shown in the top of Figure 7, and the associated standard deviations are shown in Figure 8. For the brightest stars, we achieved a differential-phase precision of $\pm 1.7 \mathrm{mrad}$.

\footnotetext{
${ }^{12}$ Removing a polynomial of order 7 is about equivalent to removing any feature with a spectral width larger than $400 \mathrm{~nm} / 6=66 \mathrm{~nm}$. This should remove most traces of dispersion without impacting photon-noise estimations and narrowband spectral features. The order was chosen to have the standard deviation of the self-calibrated average phases close to the photon-noise model.
}

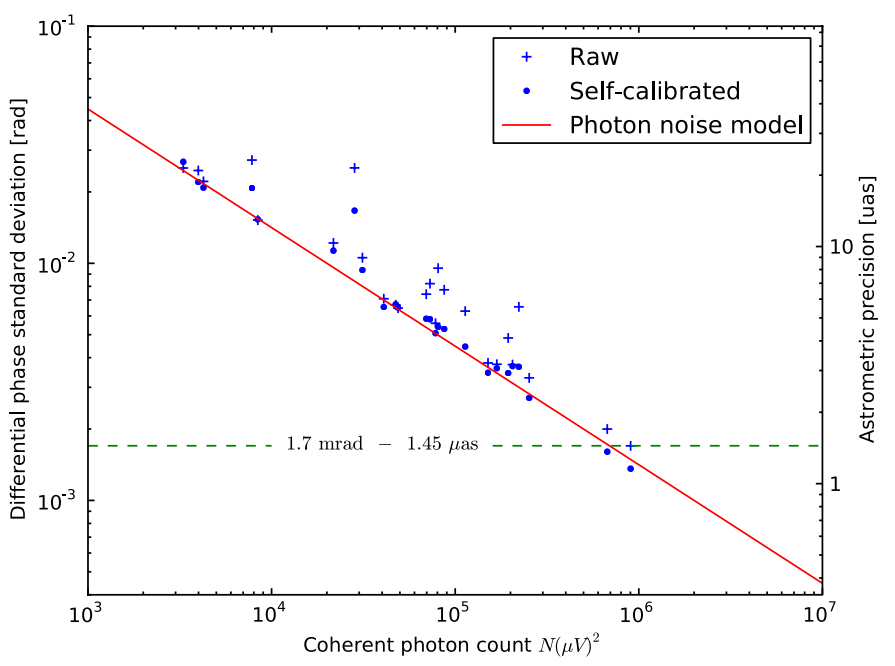

FIG. 8.-Average differential-phase standard deviation vs. total photon count. The self-calibrated average differential phase follows the overlaid photon-noise model, whereas the raw average differential phase shows a slightly higher level of noise, but without any noise floor, implying that longer data collection intervals should yield precisions better than the demonstrated $\pm 1.7 \mathrm{mrad}$ or $\pm 1.45 \mu$ as differential astrometry. See the electronic edition of the PASP for a color version of this figure.

The accuracy of the differential-phase measurement was not verified on a calibrator, mainly due to the difficulty of finding targets with known signatures that are measurable by the instrument. We cannot use known binary stars, our standard accuracy references for broadband $V^{2}$ : the field of view at $\lambda=2.2 \mu \mathrm{m}$ of the $D=10 \mathrm{~m}$ Keck telescopes is not large enough, with respect to the $B=85 \mathrm{~m}$ baseline length, for a binary to produce a differential-phase modulation shorter than the $\Delta \lambda=0.35 \mu \mathrm{m}$ wavelength coverage $(B / D \times \Delta \lambda / \lambda \sim 1.3)$. The accuracy can be inferred from the different aspects of the phase measurement, however: the amplitude and linearity of the dither, the absolute knowledge of the wavelength for each spectral channel, the linearity of the detector, the knowledge of the refractive index, and potential contamination of the spectral measurements by light coming from the undispersed channel. All these terms have been estimated to have a contribution below the demonstrated limit of $1.7 \mathrm{mrad}$. However, in order to highlight any potential bias in the differential-phase measurement versus wavelength, we also studied the best self-calibration of the data-the average difference of two consecutive measurements:

$$
\sigma_{\lambda}\left[\left\langle\Phi_{n+1}(\lambda)-\Phi_{n}(\lambda)\right\rangle_{n}-P_{7}(\lambda)\right]
$$

As for the average sum, we also subtract a best-fit polynomial. When running the spectrometer at $1 \mathrm{~Hz}$ (the slowest we have run it at for science observations), the difference of two consecutive measurements eliminates any effect that evolves on timescales shorter than $1 \mathrm{~s}$. This self-calibration is our best estimate of the impact of photon noise on the differential-phase measurement 


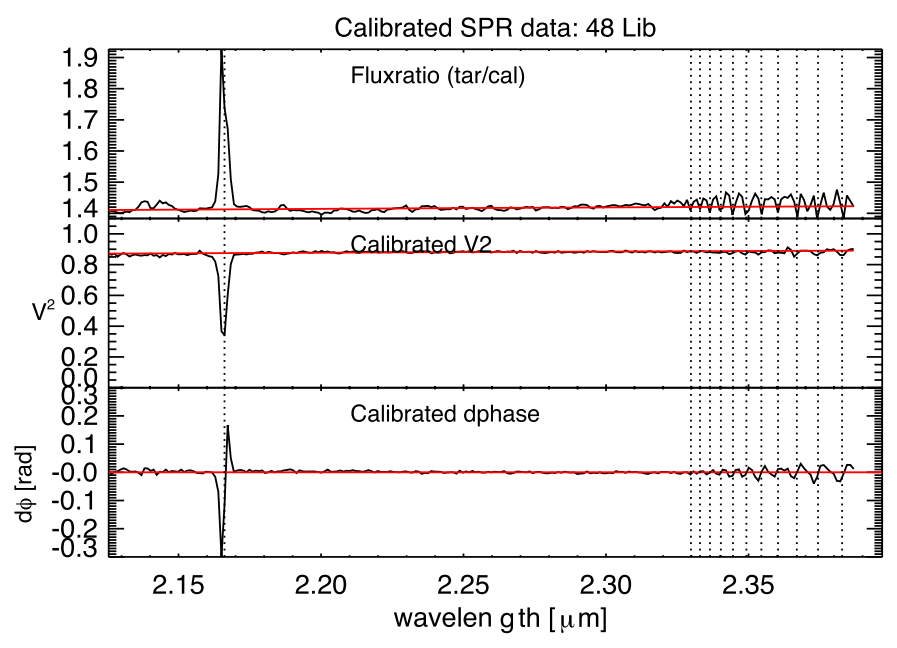

FIG. 9.-Exact copy of Fig. 1 from Pott et al. (2010), illustrating the performance of the instrument on $\mathrm{Br} \gamma$ and Pfund series emission lines from the rotating disk of the Be star 48 Lib. Top: Mean calibrated flux ratio between the $48 \mathrm{Lib}$ and the continuum-divided calibrator. The solid line marks a linear continuum fit. The different line profiles of $\mathrm{Br} \gamma$ and $\mathrm{Pf}$ emission lines are clearly visible. Center: Mean calibrated $V^{2}$ of $48 \mathrm{Lib}$ showing that both the NIR continuum and the recombination line emission are spatially resolved by the interferometer. A linear continuum (solid line) was fitted to the line free regions. Bottom: The calibrated differential-phase data. The solid line marks zero phase. All plots show the rest wavelength of the target. The vertical dotted lines indicate the rest wavelength of the recombination lines. It is apparent that all lines show the same slope at the line center, as expected for disk emission. See the electronic edition of the PASP for a color version of this figure.

(as illustrated in Fig. 8 by the better agreement between the $1 / \sqrt{N\left(\mu V^{2}\right)}$ photon-noise model and the self-calibrated data). At least for the measurements with the highest integrated coherent photon count, the agreement between the raw and selfcalibrated average differential-phase standard deviation shows that we are not impacted, at least to a precision of $\pm 1.7 \mathrm{mrad}$. Also, the absence of any visible noise floor is a good indication that we could indeed reach a better precision by accumulating more data. In addition, because we are measuring a phase, the bias sources are probably periodic with the absolute phase and could be averaged out as long as measurements are recorded at various phase track points. ${ }^{13}$ This is the path we would follow if we were limited by one of the bias terms mentioned previously.

It is also worth noting that the recommended calibration procedure for differential phase (see eq. [2]) is not imperative, as long as raw and self-calibrated processing are in agreement. The recommended procedure will, however, remove differentialphase biases below the demonstrated \pm 1.7 mrad precision and addresses most of the static air dispersion bias.

\footnotetext{
${ }^{13} \mathrm{~A}$ modulation of the phase track-point is a technique used by the $V^{2}$ mode of KI to provide an accurate visibility estimation, despite nonlinearities in the highfrequency dither. The SPR instrument uses a more accurate low-frequency dither.
}

Finally, in the limit of unresolved objects, the differential phase $\Phi(\lambda)$ is related to the differential astrometry of the object's photocenter, $\alpha(\lambda)$, projected on the interferometer baseline (Petrov et al. 1982). The accuracy on the differential phase $\delta \Phi$ can be converted into an accuracy on differential astrometry $\delta \alpha$ as follows:

$$
\frac{\delta \alpha}{|\alpha|}=\frac{\delta(B / \lambda)}{|B / \lambda|}+\frac{\delta \Phi}{|\Phi|}
$$

Involving the relative accuracy on the baseline and wavelength, this requirement is identical to the narrow-angle astrometry requirement (Shao \& Colavita 1992); however, the conditions of application are very different. A narrow-angle measurement accurate at a few-microsecond level, between two objects separated by a few arcseconds, requires a relative baseline knowledge on the order of $10^{-6}$. For differential phase, the separation of a spectral feature photocenter from a continuum photocenter is typically well under the 5 mas angular resolution of the interferometer, requiring only a relative baseline knowledge of $10^{-2}$. This is well within the baseline knowledge of the Keck interferometer. The $\pm 1.7 \mathrm{mrad}$ differential-phase precision and accuracy are therefore directly equivalent to a differential astrometry precision and accuracy of $\pm 1.45 \mu$ as.

\section{CONCLUSION}

The self-phase-referencing science mode of the Keck Interferometer provides a $K$-band spectral resolution of $R \sim 1000$ on targets as faint as $K=7.8 \mathrm{mag}$ with spatial resolution as fine as 5 mas. The $V^{2}(\lambda)$ calibration accuracy performance has been shown to be better than \pm 0.02 absolute and better than \pm 0.01 differential. The differential-phase precision and accuracy for narrow spectral features has been shown to be better than $\pm 1.7 \mathrm{mrad}$ or $\pm 1.45 \mu$ as on sky. Illustrative science results obtained with SPR can be found on a few young stellar objects in Eisner et al. (2010) or Eisner \& Hillenbrand (2011) and on a Be star in Pott et al. (2010; see Figure 9).

The limiting magnitude of this instrument could be improved, however, by investing into a 90/10 beam splitter between the fringe tracker and the spectrometer, to direct most of the light to the fringe tracker. The limiting magnitude of the instrument could then be pushed to $K=9.4$, which might be sufficient to observe the brightest active galactic nuclei. The SPR instrument could also be extended to other science wavelengths: namely, $H$ and $L$ bands, currently offered by the Keck Interferometer. In the longer term, higher spectral resolution could be offered by, for example, feeding the fiber output of the interferometer to NIRSPEC (McLean et al. 1998), an existing Keck science instrument that offers a spectral resolution of $R>20,000$. Finally, SPR provides the basis for dual-field phase-referencing, the next improvement to be provided by the ASTRA project, on the path to narrow-angle astrometry. 
The W. M. Keck Observatory is operated as a scientific partnership among the California Institute of Technology, the University of California, and the National Aeronautics and Space Administration. The Observatory was made possible by the generous financial support of the W. M. Keck Foundation. The authors wish to recognize and acknowledge the very significant cultural role and reverence that the summit of Mauna Kea has always had within the indigenous Hawaiian community. We are most fortunate to have the opportunity to conduct observations from this mountain. The Keck Interferometer is funded by the National Aeronautics and Space Administration. The ASTRA project was funded by the Major Research Instrumentation program of the National Science Foundation (grant AST-0619965). This work has made use of services produced by the NASA Exoplanet Science Institute at the California Institute of Technology.

\section{APPENDIX}

\section{ELEMENTS OF THE ERROR BUDGET}

Flux above telescopes $\left[\boldsymbol{F}_{\text {in }}\right]$.- Total flux collected by the two telescopes for a $K=10$ target.

Total transmission $[\% \boldsymbol{T}]$.-Combination of the static, turbulent (AO-corrected Strehl ratio and beam train), $K / N$ splitters, and grism transmissions.

Flux at combiner outputs $\left[\boldsymbol{F}_{\text {out }}\right]$.- Flux above telescope times total transmission. Represents the photon flux summed over the two outputs of the beam combiner, for a $K=10$ target:

$$
F_{\text {out }}=F_{\text {in }} \times \% T \text {. }
$$

One output throughput.- Standard transmission metric for the Keck Interferometer that represents the total flux available at one beam-combiner output for a $K=10$ mag star, expressed in digital unit (0.26 DN per electron). This quantity is standard diagnosis output of the data reduction pipeline, given in the error budget table as a reference.

Observable transmission $[\gamma]$.- Fraction of the total flux, from the two beam-combiner outputs, used for the estimation of the phase $\mathrm{S} / \mathrm{N}$. The fringe trackers use all the available bandpass $(\delta \lambda=350 \mathrm{~nm})$, from both beam-combiner outputs $\left(N_{\text {output }}=2\right)$. The spectrometer uses only one spectral channel $(\delta \lambda=1.1 \mathrm{~nm})$, from only one beam-combiner output $\left(N_{\text {output }}=1\right)$ :

$$
\gamma=\frac{\delta \lambda}{350 \mathrm{~nm}} \frac{N_{\text {output }}}{2}
$$

Electron per observable $[N]$.- Mean number of electrons detected from the given $K$ magnitude target, in the given integration time $t$, based on the $K=10$ flux at both combiner output $F_{\text {out }}$, and the observable transmission $\gamma$ :

$$
N=\gamma t F_{\text {out }} 10^{(10-K) / 2.5} .
$$

Fringe contrast $\left[\mu^{2}\right]$.- - Fringe contrast from the combined fringe tracker residual piston $\sigma_{\mathrm{ft}}$ and spectrometer differential piston $\sigma_{\text {spectro }}$. At a wavelength of $\lambda=2.15 \mu \mathrm{m}$, the two contributions are combined into a fringe contrast as follows:

$$
\mu^{2}=e^{-\left(\frac{2 \pi}{\lambda}\right)^{2}\left(\sigma_{\mathrm{ft}}^{2}+\sigma_{\text {spectro }}^{2}\right)} .
$$

Total noise $\left[\sigma_{N B R}\right]$ - - Combination of the photon noise $\sigma_{N}$ (estimated from the number of electrons per observable), the measured background noise $\sigma_{B}$, and the measured readout noise $\sigma_{R}$. The readout noise on the spectrometer is lower than on the fringe tracker for two reasons. (1) The spectrometer error budget takes into account only 1 pixel, whereas the fringe tracker uses 5 pixels. (2) With lower rates, the spectrometer is able to perform multiple readouts:

$$
\sigma_{N B R}^{2}=\sigma_{N}^{2}+\sigma_{B}^{2}+\sigma_{R}^{2}
$$

Phase $\mathbf{S} / \mathbf{N}[\mathbf{S} / \mathbf{N}] /$ phase error $\mathbf{r m s}\left[\sigma_{\Phi}\right]$. - Phase $\mathrm{S} / \mathrm{N}$ based on the mean number of electrons per observable, the fringe contrast, and the different noise sources, assuming a target visibility $V$ of 1, and following Walkup \& Goodman (1973):

$$
\mathrm{S} / \mathrm{N}=\frac{1}{\sigma_{\Phi}}=\sqrt{\gamma \frac{N^{2}(\mu V)^{2}}{2 \sigma_{N B R}^{2}}},
$$

where $\gamma=8 / \pi^{2}$ is the coherence loss factor from the fourquarter-wave bin phase estimator.

\section{REFERENCES}

Akeson, R., \& Millan-Gabet, R. 2005, KI Visibility Amplitude $\left(V^{2}\right)$ Data Reduction Guide (Pasadena: NExScI), http://nexsci.caltech .edu/software/KISupport/v2/V2reductionGuide.pdf

$\rightarrow$ Akeson, R. L., Swain, M. R., \& Colavita, M. M. 2000, Proc. SPIE, 4006, 321

Berio, P., et al. 1999, A\&A, 345, 203 $\rightarrow$ Boden, A. F., Torres, G., \& Latham, D. W. 2006, ApJ, 644, 1193

Colavita, M. 2001, BAAS, 33, 859

$\rightarrow$ Colavita, M., et al. 2003, ApJ, 592, L83

$\rightarrow$ Colavita, M. M. 1999, PASP, 111, 111

$\rightarrow$ Colavita, M. M., Booth, A. J., Garcia-Gathright, J. I., Vasisht, G., Johnson, R. L., \& Summers, K. R. 2010, PASP, 122, 795 
$\rightarrow$ Colavita, M. M., Serabyn, G., Wizinowich, P. L., \& Akeson, R. L. 2006, Proc. SPIE, 6268, 626803

$\rightarrow$ Colavita, M. M., et al. 2008, Proc. SPIE, 7013, 70130A

$\rightarrow$. 2009, PASP, 121, 1120

$\rightarrow$ Delplancke, F., et al. 2006, Proc. SPIE, 6268, 62680U

Eisenhauer, F., Perrin, G., Rabien, S., Eckart, A., Lena, P., Genzel, R., Abuter, R., \& Paumard, T. 2005, Astronomische Nachrichten, 326, 561

Eisner, J. A., \& Hillenbrand, L. A. 2011, ApJ, in press

$\rightarrow$ Eisner, J. A., et al. 2010, ApJ, 718, 774

$\rightarrow$ Gai, M., et al. 2004, Proc. SPIE, 5491, 528

$\rightarrow$ Le Bouquin, J.-B., Bauvir, B., Haguenauer, P., Schöller, M., Rantakyrö, F., \& Menardi, S. 2008, A\&A, 481, 553

$\rightarrow$ McLean, I. S., et al. 1998, Proc. SPIE, 3354, 566

$\rightarrow$ Millour, F., et al. 2007, A\&A, 464, 107

$\rightarrow$ Monnier, J. D., et al. 2008, Proc. SPIE, 7013, 701302

$\rightarrow$ Mourard, D., Bosc, I., Labeyrie, A., Koechlin, L., \& Saha, S. 1989, Nature, 342, 520

$\rightarrow$ Mourard, D., et al. 2009, A\&A, 508, 1073 $\rightarrow$ Perrin, G. 2003, A\&A, 400, 1173

$\rightarrow$ Petrov, R., Kadiri, S., Martin, F., Ricort, G., \& Aime, C. 1982, J. Opt., 13, 331

$\rightarrow$ Petrov, R. G., et al. 2007, A\&A, 464, 1

$\rightarrow$ Pott, J., et al. 2010, ApJ, 721, 802

$\rightarrow$ Quirrenbach, A., et al. 1998, Proc. SPIE, 3350, 807

$\rightarrow$ Ragland, S., et al. 2008, Proc. SPIE, 7013, 70130B

$\rightarrow$ Schmitt, H.R., et al. 2009, ApJ, 691, 984

Shao, M., \& Colavita, M. M. 1992, A\&A, 262, 353

$\rightarrow$ Sturmann, J., Ten Brummelaar, T., Sturmann, L., \& McAlister, H. A. 2010, Proc. SPIE, 7734, 77343A

Vakili, F., Mourard, D., Bonneau, D., Morand, F., \& Stee, P. 1997, A\&A, 323, 183

Vakili, F., et al. 1998, A\&A, 335, 261

$\rightarrow$ Vasisht, G., Booth, A. J., Colavita, M. M., Johnson, R. L., Jr., Ligon, E. R., III, Moore, J. D., \& Palmer, D. L. 2003, Proc. SPIE, 4838, 824

$\rightarrow$ Walkup, J. F., \& Goodman, J. W. 1973, J. Opt. Soc. Am., 63, 399

$\rightarrow$ Wizinowich, P., et al. 2000, PASP, 112, 315

$\rightarrow$ Woillez, J., et al. 2010, Proc. SPIE, 7734, 773412 